\title{
Estimating the Rotational Synchronous Component from Instantaneous Angular Speed Signals in Variable Speed Conditions
}

\author{
Guillaume Bruand ${ }^{12}$, Florent Chatelain ${ }^{1}$, Pierre Granjon ${ }^{1}$, Nadine Martin ${ }^{1}$, \\ Christophe Duret $^{2}$, and Hervé Lénon ${ }^{2}$ \\ ${ }^{1}$ Univ. Grenoble Alpes, CNRS, Grenoble INP*, GIPSA-lab, \\ 38000 Grenoble, France \\ ${ }^{2}$ NTN-SNR Roulements, 74000 Annecy, France \\ *Institute of Engineering Univ. Grenoble Alpes \\ guillaume.bruand@gipsa-lab.grenoble-inp.fr
}

\begin{abstract}
Condition monitoring performed directly from the estimated instantaneous angular speed has found some interesting applications in industrial environments, going from bearing monitoring to gear failure detection. One common way to estimate the angular speed makes use of angular encoders linked to a rotating shaft. At the opposite of traditional time-sampled signals, encoders describe purely angular phenomena often encountered in rotating machines. However, rotating encoders suffer from various geometric defects, corrupting the measurement with an angular periodic signature. The angular synchronous average is a very popular tool to estimate this systematic error, but is only adapted to constant speed conditions, which is rarely the case in real applications. We propose here two different estimators to compute a robust estimation of the synchronous component in variable speed conditions. The former, as a data-driven approach, is based on a local weighted least squares method, while the latter is a model-based approach. We study the behaviour of our estimators with both simulations and experimental signals, and show the relevance of the proposed method in an industrial context.
\end{abstract}

Keywords: Condition monitoring · Instantaneous Angular Speed · Synchronous average $\cdot$ Non-stationary

\section{Introduction}

The use of Instantaneous Angular Speed (IAS) for condition monitoring is an interesting alternative to more popular tools, such as vibration analysis or acoustic emissions. It has been applied in various industrial cases involving rotating parts, such as wind turbines [2], engines [9, 11, 12], bearings [8], gearboxes [10] or industrial machines [6]. One of the main feature of IAS is the angular localization of the measurement, which gives an insight in common angle-linked phenomena encountered when dealing with rotating machines. One technique to estimate the IAS is to associate a high resolution angle measurement with a high frequency 
clock. The angle measurement can be achieved thanks to the combination of a rotating encoder, such as zebra tapes or magnetic encoder, with the appropriate sensor.

These types of encoders need calibration to deal with various sources of errors, such as irregularity of tapes or distortion of the measured magnetic field, both corrupting the angle estimation with repeatable error. However it is not always possible to calibrate the sensor with the help of a reference angle. The processings proposed in this paper address the case where no reference angle is available.

One of the most popular tool to deal with this kind of repeatable error is the Residual Angle Synchronous Average, which is the angular equivalent to the Time Synchronous Average. A first step processes the estimated IAS by synchronous averaging, and then the synchronous part identified as the undesired error is removed. However, the synchronous average does not cope with variable speed conditions, as we will demonstrate further. In [1] a Generalized Synchronous Average is proposed, computing the synchronous average at different regimes and using a kernel smoother to give a continuous speed-dependant synchronous estimation. However, this kind of data-driven approach is limited by the number of cycles needed to obtain a proper average for each regime. In this paper we present two different approaches to retrieve a synchronous component in variable speed, both based on a model developed in section 2. Section 3 presents local polynomial regression, a useful tool to deal with IAS measurements. Section 4 introduces some notations relating to the synchronous average operator, while sections 5 and 6 detail the two aforementioned methods to estimate the synchronous component. Finally simulation results are given in section 7.

\section{The Instantaneous Angular Speed Measurement}

In this study we consider an angle measurement obtained from a rotating encoder and the paired sensor. One popular technology is the zebra-tapes encoder, associated with an optical sensor, where white and black tapes are used alternatively to give the angular position. The magnetic encoder is a robust alternative, where the angular position is obtained from the magnetic field emitted by alternation of magnetic poles of opposite polarity. This kind of measurement is directly linked to the angular position of the shaft, with a given angular resolution often referred as a number of points per revolution.

The measurement of IAS is presented in [7], where both an elapsed-time method and a pulse counter method are detailed. The approach used in this paper is the elapsed-time method, where a high frequency clock is used to measure the elapsed time between two pulses given by the angular encoder.

For now we consider a rotating shaft, which angular position (given by some encoder) is known as $\theta(t)$. The rotating speed is noted $\omega(t)=\frac{\mathrm{d} \theta}{\mathrm{d} t}$. We suppose that the shaft never stops, i.e. $\forall t, \omega(t)>0$. So there is a bijection between 
the angular and time domains: when the angle is given as function of time, say $\theta=\varphi(t)$, the time is written equivalently as function of angle, which is written $t=\varphi^{-1}(\theta)$. In the angle domain, the rotating speed can also be given for each angular position $\theta$, with the equivalence $\omega(t)=\omega\left(\varphi^{-1}(\theta)\right)=\Omega(\theta)$. In fact this representation is quite natural in this application, where the encoder is physically linked to the rotating part: the elapsed time is given between fixed angle positions.

The time $t$ can be directly expressed by integrating the rotating speed,

$$
t(\theta)=\int_{0}^{\theta} \frac{\mathrm{d} \varphi}{\Omega(\varphi)},
$$

and thus deriving the time allows one to retrieve the instantaneous angular speed $\Omega(\theta)$.

The angle is sampled with a regular step $\Delta \theta$, such that we obtain a set of measurement $\left(\theta_{n}\right)_{n \in \mathbb{N}}=(n \Delta \theta)_{n \in \mathbb{N}}$. A classical approach is to differentiate the previous equation $\Delta t_{n}=t\left[\theta_{n+1}\right]-t\left[\theta_{n}\right]$, which in practice is called the "elapsed time method". The main drawback of this method (or similar ones based on finite differentiation) is that differentiation is very sensitive to white noise, as high frequencies would overwhelm the signal.

A different approach is developed here, based on local polynomial approximation. Intuition is the following: for a constant speed $\Omega(\theta)=\Omega_{0}$, integration in eq.(1) leaves a linear term

$$
t(\theta)_{\Omega=\Omega_{0}}=\frac{\theta}{\Omega_{0}} .
$$

More generally, we can write

$$
t(\theta)=l(\theta)+h(\theta)
$$

as the sum of two components, one component $l(\theta)$ containing the very low frequencies (for example, the trend in eq.(2)) and a second component $h(\theta)=$ $t(\theta)-l(\theta)$ containing the remaining high frequencies. We will discuss about the discrimination between low and high frequencies later.

Let us now introduce the sampling errors corrupting the measurement. At each angular position $\theta_{n}$, a repeatable, deterministic error $\xi_{n}$ is committed due to the inner characteristics of the encoder, typically pole irregularities, or distortion of the magnetic field. The sampling angles are noted

$$
\overline{\theta_{n}}=\theta_{n}+\xi_{n} .
$$

We assume that $\xi_{n}$ is periodic over one revolution, i.e. when the measurement is sampled at $M \in \mathbb{N}$ points per revolution, we have $\forall n, \xi_{n+M}=\xi_{n}$. The expression of the corresponding time $t\left[\overline{\theta_{n}}\right]$ is obtained from eq.(3),

$$
t\left[\overline{\theta_{n}}\right]=l\left[\overline{\theta_{n}}\right]+h\left[\overline{\theta_{n}}\right] .
$$


For a small error $\xi_{n}$ and a sufficiently smooth speed, i.e. the speed does not vary too much regarding the sampling frequency, a first order approximation gives

$$
t\left[\overline{\theta_{n}}\right]=t\left[\theta_{n}\right]+\xi_{n} \frac{\mathrm{d} t}{\mathrm{~d} \theta}\left[\theta_{n}\right]=l\left[\theta_{n}\right]+h\left[\theta_{n}\right]+\xi_{n} t^{\prime}\left[\theta_{n}\right]
$$

where we have introduced $t^{\prime}\left[\theta_{n}\right]=\frac{\mathrm{d} t}{\mathrm{~d} \theta}\left[\theta_{n}\right]$. We now see that the measurement is corrupted with the periodic error $\xi_{n}$. The idea developed in this paper is to:

1. Get a smooth estimate of the speed $\Omega$ thanks to the polynomial approximation $l(\theta)$

2. Estimate the repeatable angular error $\xi_{n}$

3 . Use both of these informations to correct the original sampling.

\section{A Local Polynomial Regression}

The objective in this section is to estimate the low frequency content of the time signal $t\left[\overline{\theta_{n}}\right]$, noted $l\left[\overline{\theta_{n}}\right]$ in eq.(5). We fit $t\left[\overline{\theta_{n}}\right]$ locally with a polynomial of low order, a common method described as "lowess smoothing" in [4]. Let us explain this choice in the scope of our study.

1. When the speed is constant, the "very low frequency component" is just an affine function (see eq.(2)). It makes sense to use linear regression.

2. In real cases, when speed is slowly varying, the corresponding recorded time $t\left[\overline{\theta_{n}}\right]$ can be locally approximated by a low order polynomial, such as a line or a parabola. The "local" property is determined by a tuning parameter used to cope with different speed profiles.

3 . Estimating the time $t\left[\overline{\theta_{n}}\right]$ with a piecewise polynomial gives a smooth estimate of the speed, free from measurement noise

4. Moreover the residual obtained by removing the very low frequency content is not distorted and can be used for further analysis

As the local polynomial fit has already been investigated in the scope of IAS estimation in [13], we will not discuss the detailed performances of the method here. However, we recall the principle of this estimation as we use a similar idea in section 5:

- The signal $t\left[\overline{\theta_{n}}\right]$ is fitted with a polynomial of low order $K$, hence the linear model

$$
\mathbf{t}_{n}=X \boldsymbol{\beta}_{n}+\mathbf{r}_{n}
$$

where at sample $n \in \mathbb{N}$, the local span of size $N=2 P+1$ of the signal is noted $\mathbf{t}_{n}=\left[t\left[\overline{\theta_{n-P}}\right] \ldots t\left[\overline{\theta_{n+P}}\right]\right]^{T}, \boldsymbol{\beta}_{n} \in \mathbb{R}^{K+1}$ are the coefficients to be estimated, $\mathbf{r}_{n}$ is the residual, and $X$ is the constant design matrix used to estimate the low frequencies $l\left[\theta_{n}\right]$ sampled at regularly spaced angles $\theta_{n}=n \Delta \theta$, such as 


$$
X=\left[\begin{array}{cccc}
1 & 1 & \ldots & 1 \\
1 & 2 & \ldots & 2^{K} \\
\vdots & \vdots & \vdots & \vdots \\
1 & N & \ldots & N^{K}
\end{array}\right] \in \mathbb{R}^{N \times(K+1)}
$$

- The least square estimate of coefficients $\hat{\boldsymbol{\beta}}_{n} \in \mathbb{R}^{K+1}$ is given by

$$
\hat{\boldsymbol{\beta}}_{n}=\underset{\boldsymbol{\beta}}{\arg \min }\left(\mathbf{t}_{n}-X \boldsymbol{\beta}\right)^{T} W\left(\mathbf{t}_{n}-X \boldsymbol{\beta}\right)
$$

with $W$ a weighting matrix:

$$
W=\left[\begin{array}{cccc}
w_{1} & 0 & \cdots & 0 \\
0 & w_{2} & & \vdots \\
\vdots & & \ddots & 0 \\
0 & \cdots & 0 & w_{N}
\end{array}\right] \in \mathbb{R}^{N \times N}
$$

where the weights $\left(w_{i}\right)_{i \in 1 . . N}$ privilege samples near the current one; for example the popular tricube function described in [4] by

$$
\forall i \in\{1 \ldots N\}, \quad w_{i}=\left(1-\frac{|P-i|^{3}}{P^{3}}\right)^{3} .
$$

A simple derivation along $\boldsymbol{\beta}$ in eq.(8) gives the well known formula

$$
\hat{\boldsymbol{\beta}}_{n}=\left(X^{T} W X\right)^{-1} X^{T} W \mathbf{t}_{n} .
$$

- The residual is computed from the middle of the fit $X \hat{\boldsymbol{\beta}}_{n}$, which is given by the $P^{t h}$ sample $r\left[\overline{\theta_{n}}\right]=t\left[\overline{\theta_{n}}\right]-\left(1 P \ldots P^{K}\right) \hat{\boldsymbol{\beta}}_{n}$.

The fit gives a noise free estimation of the signal $t\left[\overline{\theta_{n}}\right]$, approximated locally by a polynomial $\hat{l}\left[\theta_{n}\right]$ at the center of the span,

$$
\forall n \in \mathbb{N}, \quad \hat{l}\left[\theta_{n}\right]=\left(\begin{array}{lll}
1 & P \ldots P^{K}
\end{array}\right) \hat{\boldsymbol{\beta}}_{n}
$$

and a residual, obtained from 6 by a simple substraction

$$
r\left[\overline{\theta_{n}}\right]=t\left[\overline{\theta_{n}}\right]-\hat{l}\left[\theta_{n}\right] \simeq h\left[\theta_{n}\right]+\xi_{n} t^{\prime}\left[\theta_{n}\right] .
$$

The least squares estimator $\hat{\boldsymbol{\beta}}_{n}$ in eq.(9) shows that the local polynomial regression can be viewed as a linear filter of span $N$. Thus the expression of $r\left[\overline{\theta_{n}}\right]$ in (11) is just a high-passed filtered version of $t\left[\overline{\theta_{n}}\right]$. The order $K$ of the polynomial and the span $N$ are chosen according to the specifications of the user, determining the "local" nature of the fit, or equivalently the cut-off frequency the filter (see fig. 1).

We present two different ways to estimate the error $\xi_{n}$ : the first one presented in section 5 is a data-driven approach, where $\xi_{n}$ is used as a local regressor to 


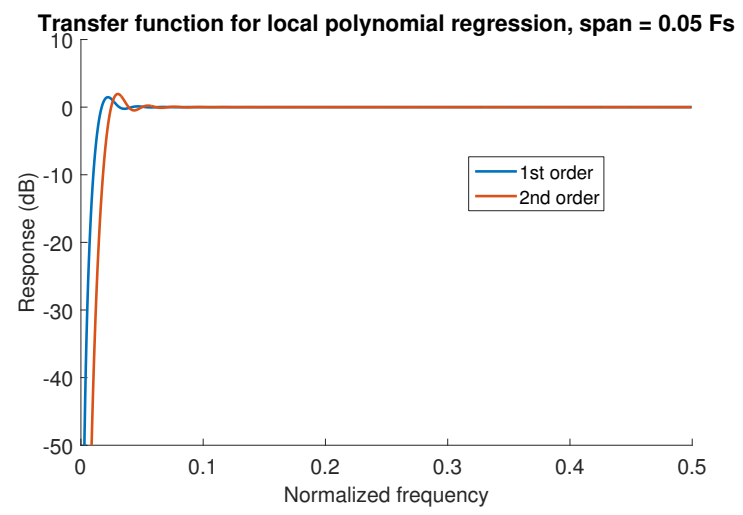

Fig. 1: The transfer function associated to the polynomial regression filter described in eq.(11), comparison between polynomials of orders $K=1$ and $K=2$

estimate the modulation $t^{\prime}\left[\theta_{n}\right]$. The second one presented in section 6 is a simple derivation of the angular error $\xi_{n}$ using the filtered version of the time $\hat{l}\left[\theta_{n}\right]$ (see eq.(10)). Both of our methods rely on the synchronous average operator, a tool presented in the following section.

\section{The Angle Synchronous Average}

The synchronous average is a popular tool dealing with periodic components. Let us introduce some notations describing the associated operator.

\subsection{The Synchronous Average Operator}

We note $M \in \mathbb{N}^{*}$ the number of points per revolution, a.k.a. the resolution of the encoder. One central operation in the following parts is the synchronous average, which is an average over several revolutions. We define for every discrete signal $s$ the synchronous averaging operator

$$
\forall k \in\{1 \ldots M\}, \quad m_{s}[k]=\lim _{T \rightarrow+\infty} \frac{1}{T} \sum_{p=1}^{T} s[k+p M] .
$$

Note that for $M=1, m_{s}$ is simply the mean operator. The synchronous average $m_{s}$ is a vector of $\mathbb{R}^{M}$; the operator is linear, and for a $M$-periodic signal $\tilde{s}$ we have

$$
\forall k \in\{1 \ldots M\}, \quad m_{\tilde{s}}[k]=\tilde{s}[k] .
$$

The main property of the synchronous average is that it only captures components which frequencies are integer orders: in the frequency domain, the synchronous average operator can be seen as a comb filter at every integer orders[3]. 
Using the linearity of the synchronous average operator, a direct consequence is that every signal can be written as a sum of two components, the synchronous average and the non-synchronous component $v$ in which every integer frequency have been removed,

$$
s=m_{s}+v \text {. }
$$

\subsection{Separating Synchronous Components}

In fact the synchronous average cannot be directly applied on the raw time signal $t\left[\overline{\theta_{n}}\right]$. Indeed it would be biased due to the fact that it is a finite duration signal, containing a trend and low frequencies that would not be averaged to vanish in the synchronous estimation.

For this reason we use the residual $r\left[\overline{\theta_{n}}\right]$ obtained after the local polynomial fit presented in the previous section (see eq.(11)), which behaves like a high pass filter. In the following paragraphs, we present two different approaches to get rid of the periodic error $\xi_{n}$ in the raw signal.

Since $h$ is the high frequency content of $t$ (see eq.(6)) it is centered, and to simplify the interpretation we use the hypothesis that it does not contain any synchronous component, both conditions required to have $m_{h}\left[\theta_{n}\right]=0$.

In this case we can apply the synchronous average to eq.(11), and using the linearity of the operator and the $M$-periodicity of $\xi_{n}$ we get

$$
m_{r}\left[\overline{\theta_{n}}\right]=\underbrace{m_{h}\left[\theta_{n}\right]}_{=0}+\xi_{n} m_{t^{\prime}}\left[\theta_{n}\right]=\xi_{n} m_{t^{\prime}}\left[\theta_{n}\right] .
$$

Note that $t$ being a time counter, we have $t^{\prime}(\theta)>0$ and thus $m_{t^{\prime}}\left[\theta_{n}\right]>$ 0 . In real cases the synchronous components are not separated from the error $\xi_{n} t^{\prime}\left[\theta_{n}\right]$, this is why any attempt to correct the periodic error will also remove the synchronous components.

\section{Synchronous Regression}

The first proposed estimator uses the fact that the periodic error $\xi_{n}$ is modulated in amplitude by the term $t^{\prime}\left[\theta_{n}\right]$ (see eq.(11)). In the vibration analysis scope, Daher[5] has made some attempt to compute a synchronous average in variable speed with a least square approach. However a polynomial basis is used to describe the amplitude modulation of the synchronous average, while we rely on our first order model and use the synchronous average as the only predictor for the least square estimation.

The idea is then to use a synchronous average on the residual $r\left[\overline{\theta_{n}}\right]$ as a local linear fit. The model adopted is very similar to the first polynomial fit presented in section 3, except that we replace the polynomial basis expansion with the synchronous average $m_{r}\left[\overline{\theta_{n}}\right]$ as the regressor. This time we use a vector of span $M$ for each sample $n \in \mathbb{N}^{*}$, noted 


$$
\overline{\mathbf{r}}_{n}=\left[r\left[\overline{\theta_{n-P}}\right] \ldots r\left[\overline{\theta_{n+P}}\right]\right]^{T}
$$

where for sake of simplicity, we have assumed a odd number of samples $M=2 P+1$ per revolution.

Looking at eq.(15) we notice that $\xi_{n}$ is proportional to $m_{\overline{\mathbf{r}}_{n}}$, which leads us to the local model

$$
\overline{\mathbf{r}}_{n}=Y \boldsymbol{\alpha}_{n}+\mathbf{h}_{n}
$$

where

$$
\boldsymbol{\alpha}_{n} \in \mathbb{R}^{2}, Y=\left[\begin{array}{cc}
1 & m_{r}\left[\overline{\theta_{n-P}}\right] \\
1 & m_{r}\left[\overline{\theta_{n-P+1}}\right] \\
\vdots & \vdots \\
1 & m_{r}\left[\overline{\theta_{n+P}}\right]
\end{array}\right] \in \mathbb{R}^{N \times 2}
$$

Note : we have considered $m_{r}\left[\overline{\theta_{k}}\right]$ for any $k \in \mathbb{N}^{*}$ by using its value modulo $M$, and used the column of 1 in $Y$ as an intercept.

Using the same weighting matrix $W$ as in section 3 , with weights defined by the tricube function privileging samples at the center of the span, we minimize the least square weighted loss function

$$
\hat{\boldsymbol{\alpha}}_{n}=\underset{\boldsymbol{\alpha}}{\arg \min }\left(\overline{\mathbf{r}}_{n}-Y \boldsymbol{\alpha}\right)^{T} W\left(\overline{\mathbf{r}}_{n}-Y \boldsymbol{\alpha}\right) .
$$

The same formula is obtained to estimate the coefficients

$$
\hat{\boldsymbol{\alpha}}_{n}=\left(Y^{T} W Y\right)^{-1} Y^{T} W \overline{\mathbf{r}}_{n} .
$$

Finally we compute the residual from the center of the fit $Y \hat{\boldsymbol{\alpha}}_{n}$, which is given by

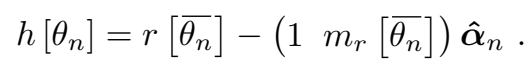

\section{Direct Estimation of the Synchronous Component}

A direct way to obtain the residual $r\left[\theta_{n}\right]$ is to estimate the angular error $\xi_{n}$. In the second method that we propose, we simply use eq.(15) to remark that

$$
\xi_{n}=\frac{m_{r}\left[\overline{\theta_{n}}\right]}{m_{t^{\prime}}\left[\theta_{n}\right]} .
$$

We need to get an estimation of $t^{\prime}\left[\theta_{n}\right]$, which is homogenous to an inverse of a speed. It seems that we are back to the initial problem, where we had to estimate an instantaneous speed from noisy measurement; however here we got a low pass filtered version of the time signal $t$, thanks to the polynomial fit presented in section 3 . The fit gave us a noise free estimation $\hat{l}\left[\theta_{n}\right]$ that we can differentiate, for example 


$$
t^{\prime}\left[\theta_{n}\right] \simeq \frac{\hat{l}\left[\theta_{n+1}\right]-\hat{l}\left[\theta_{n-1}\right]}{2 \Delta \theta} .
$$

The main advantage of this method is that it provides us directly an estimation of the error pattern $\xi_{n}$.

The corrected signal is then

$$
h\left[\theta_{n}\right]=r\left[\overline{\theta_{n}}\right]-\xi_{n} t^{\prime}\left[\theta_{n}\right] .
$$

\section{Simulation}

In order to compare the different approaches presented in this paper, we have simulated the output of an encoder rotating at variable speed. The object of the study being the estimation of the angular error caused by the encoder, we have simulated a pattern of error periodic over one revolution (figure 2a). A magnetic encoder has been considered, the angular error being caused by both the pole irregularities and the distortion of the magnetic field. The given error must be divided by the number of pole pairs to obtain the overall angular error.

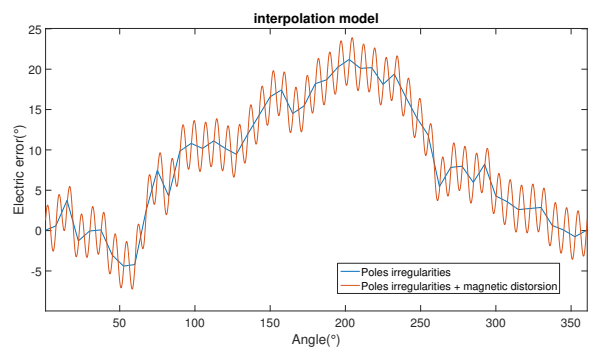

(a) Typical angular error committed using a 48 pole pairs magnetic encoder. In blue, the error due to poles irregularities, in red the contribution of the magnetic distortion.

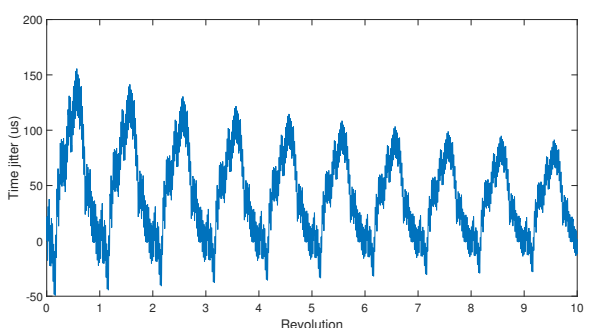

(b) The time jitter obtained with the simulated periodic error given fig.2a. Only the first 10 revolutions have been drawn.

Fig. 2: An error pattern used to simulate the time jitter polluting the speed measurement; the jitter is modulated in amplitude as the speed is growing.

A constant acceleration has been simulated, the speed varying from $500 \mathrm{rpm}$ to $5500 \mathrm{rpm}$ in $10 \mathrm{~s}$. The sampling frequency has been set to $F s=100 \mathrm{kHz}$ to avoid any distortion, and the angle has been computed by simple integration.

The speed measurement is finally obtained thanks to the elapsed-time method. On fig. $2 \mathrm{~b}$ we have computed the time jitter obtained by subtracting the time corresponding to ideal positions $\theta_{n}$. We can observe a periodic pattern linked to the angular error, and modulated in amplitude as stated in eq.(6). 
The classical synchronous average is not able to capture the jitter in this context (fig. 3): the pattern modulated in amplitude by the speed variations is not periodic and thus the residual component is still polluted. In another hand, both synchronous regression (in red) and model based approach (in yellow) result in a very small residue.
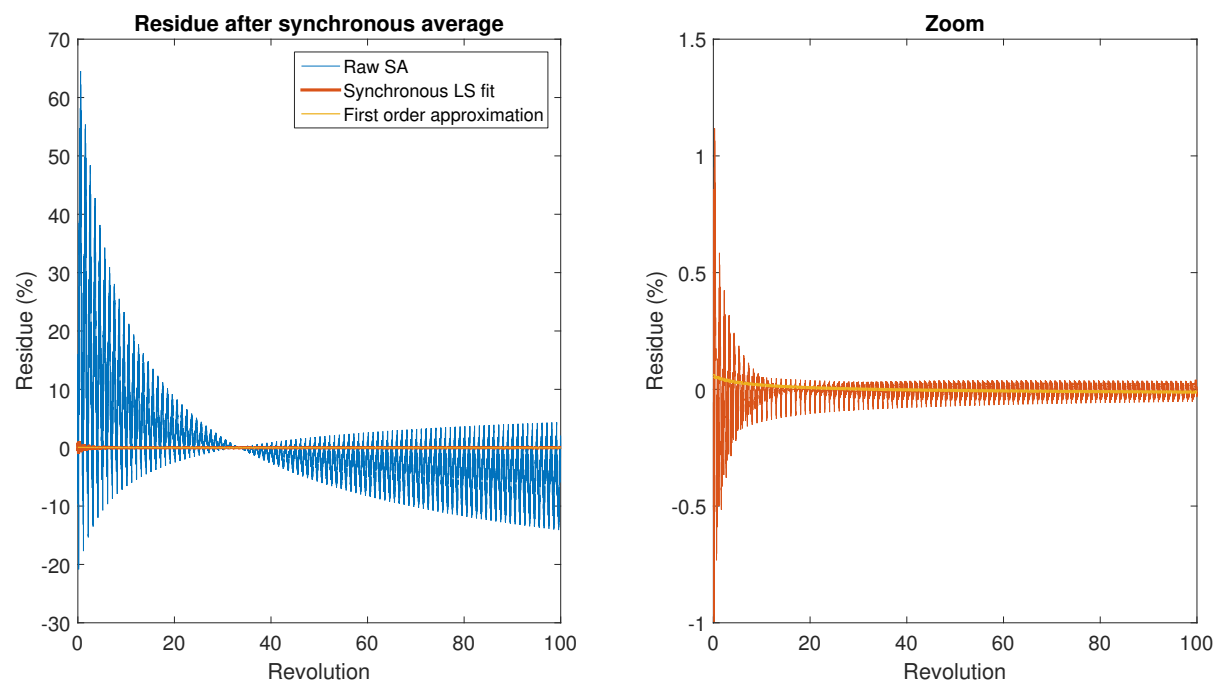

Fig. 3: The residual obtained after synchronous averaging : in blue the classical $\mathrm{SA}$, in red the synchronous regression presented in section 5 and in yellow the model based approach presented in section 6 . Results are given as a percentage of the maximum time jitter introduced in fig. $2 \mathrm{~b}$.

\section{Conclusion}

We have presented two methods to estimate the synchronous component in Instantaneous Angular Speed signals. Contrary to the classical synchronous average, these methods cope with the variable speed case. They have been used in this study to estimate and correct the periodic errors introduced by a rotating encoder. However, due to their averaging principle, these approaches do not discriminate components of same frequency (or multiple of the same frequency).

The advantages of these methods are their robustness and their simplicity, relying on very few parameters : the span width is the main tunable parameter, and it is directly interpretable as a cut off frequency. Thus they are a privileged tool in an industrial context.

One perspective could be to adapt the tuning of these parameters automatically, minimizing some given criterion on the data. Standard methods like cross-validation would then give the optimal parameters for each dataset. 


\section{Bibliography}

[1] Abboud D, Antoni J, Sieg-Zieba S, Eltabach M (2016) Deterministicrandom separation in nonstationary regime. Journal of Sound and Vibration 362(Supplement C):305 - 326, DOI https://doi.org/10.1016/j.jsv.2015. 09.029

[2] André H, Bourdon A, Rémond D (2012) Instantaneous angular speed monitoring of a $2 \mathrm{mw}$ wind turbine using a parametrization process. In: Condition Monitoring of Machinery in Non-Stationary Operations, Springer, pp 415423

[3] Braun S (2011) The synchronous (time domain) average revisited. Mechanical Systems and Signal Processing 25(4):1087 - 1102, DOI https: //doi.org/10.1016/j.ymssp.2010.07.016

[4] Cleveland WS (1979) Robust locally weighted regression and smoothing scatterplots. Journal of the American statistical association 74(368):829836

[5] Daher Z, Sekko E, Antoni J, Capdessus C, Allam L (2010) Estimation of the synchronous average under varying rotating speed condition for vibration monitoring. In: Proceedings of ISMA

[6] Lamraoui M, Thomas M, El Badaoui M, Girardin F (2014) Indicators for monitoring chatter in milling based on instantaneous angular speeds. Mechanical Systems and Signal Processing 44(1):72-85

[7] Li Y, Gu F, Harris G, Ball A, Bennett N, Travis K (2005) The measurement of instantaneous angular speed. Mechanical Systems and Signal Processing 19(4):786-805

[8] Renaudin L, Bonnardot F, Musy O, Doray J, Rémond D (2010) Natural roller bearing fault detection by angular measurement of true instantaneous angular speed. Mechanical Systems and Signal Processing 24(7):1998-2011

[9] Rivola A, Troncossi M (2014) Zebra tape identification for the instantaneous angular speed computation and angular resampling of motorbike valve train measurements. Mechanical Systems and Signal Processing 44(12):5 - 13, DOI http://dx.doi.org/10.1016/j.ymssp.2012.11.009

[10] Stander C, Heyns P (2005) Instantaneous angular speed monitoring of gearboxes under non-cyclic stationary load conditions. Mechanical Systems and Signal Processing 19(4):817-835

[11] Yang J, Pu L, Wang Z, Zhou Y, Yan X (2001) Fault detection in a diesel engine by analysing the instantaneous angular speed. Mechanical systems and signal processing 15(3):549-564

[12] Yousef Ben Sasi A, Gu F, Payne B, Ball A (2004) Instantaneous angular speed monitoring of electric motors. Journal of Quality in Maintenance Engineering 10(2):123-135

[13] Zhao M, Jia X, Lin J, Lei Y, Lee J (2018) Instantaneous speed jitter detection via encoder signal and its application for the diagnosis of planetary gearbox. Mechanical Systems and Signal Processing 98:16-31 